\title{
Faculty-Coached, Team-Based, In-Class Problem Solving in a Systematic Ap- proach Toward Undergraduate Dynamics
}

\section{Prof. Alan Bowling, University of Texas, Arlington}

Prof. Alan Bowling is from Austin, Texas and obtained his Bachelor's degree in Aerospace engineering from the University of Texas at Austin in 1988. After graduating he worked for McDonnell Douglas Space Systems Company in Houston, Texas for two years before going to graduate school at Stanford University and obtaining a Masters degree as well as a Ph. D. in Mechanical engineering in 1998. After graduation he pursued entrepenuerial activities in California for about three years. He joined the faculty at the University of Notre Dame in 2001 and moved to The University of Texas at Arlington in 2008. Prof. Bowling's interests lie in the areas of multibody dynamics, design, and control with a focus in robotic legged locomotion, as well as biomechanics at different time scales.

\section{Mr. Ashley Guy}

Ashley Guy is a doctoral student with the Robotics, Biomechanics, and Dynamic Systems Laboratory at the University of Texas at Arlington. He is currently pursuing his Ph.D. with Dr. Alan Bowling. His research includes micro- and nano-scale dynamics.

Frasier Jones, University of Texas, Arlington

Dr. Maria Adamuti-Trache, University of Texas, Arlington 


\title{
Faculty-Coached, Team-Based, In-Class, Problem Solving in a Systematic Approach Toward Undergraduate Dynamics
}

\begin{abstract}
This paper describes a new tool in active and participative learning that effectively teaches theory and practice in undergraduate dynamics using in-class problem solving. The proposed approach treats the entire class as a team while ensuring that all students participate in the problem-solving exercise. This approach addresses three key components that are known to be effective techniques in teaching and learning: 1) student engagement, 2) affective pedagogy, and 3) class management and assessment. As in other approaches toward faculty-coached in-class problem solving, it is built upon a certain restructuring of the course material into a systematic approach toward analysis and modeling of dynamic systems. The approach uses direct questioning, which has been shown to be particularly successful with female students in several different contexts. This paper presents discusses how to implement this approach in a sophomore level dynamics course.
\end{abstract}

\section{Introduction}

This paper describes a new technique to add to the arsenal of tools in the active and participative learning toolbox. This technique is similar to faculty-coached, in-class problem solving (FCICPS) ${ }^{37}$. A key component of FCICPS is that the faculty member(s) prompt and guide smaller student groups in learning and applying just taught concepts while they complete a problem in class. The goal is to attain the benefits of group work while providing just in time coaching to address any conceptual issues as they occur. Although group or team-based learning has its advantages, it still has well-known disadvantages. The main drawback being the student who is not participating may have less effective learning experiences as compared to the more gregarious and/or confident students who actually solve the problem. The purpose of this paper is to discuss this technique in the context of teaching undergraduate dynamics, although we believe it can be applied in other undergraduate science courses.

The proposed technique eliminates the small groups. The entire class solves the problem as one team. This technique has worked well for classes of up to 60 students or less; larger numbers have not yet been attempted. If done correctly, it is possible to obtain participation 
from every student in the class. It is also possible to assess the knowledge retention of each student individually in class while achieving the benefits of group work. However, the key is to engage as many students as possible in the lecture.

The approach has two key elements: 1) establish a clear structure and process for analyzing dynamic systems, and 2) directly question each student in its application to problem solving. The structure ensures that all students know the sequence of steps involved in solving a problem. The direct questioning ensures that every student participates in problemsolving, which is something that cannot be guaranteed in group work. The next sections discuss the two key elements of the proposed technique, including the authors' observations of its effectiveness. The goal is to perform a more formal investigation of these observations in the future. The purpose of this paper is to introduce and discuss the technique.

\section{Structure Behind Analyzing A Dynamic System}

The proposed approach relies on a clear structure that consists of several simple steps to follow in analyzing the motion of dynamic systems. Figure 1 shows a flow chart that illustrates the general approach ${ }^{5}$. The process in Figure 1 defines a general strategy for analyzing dynamic systems that allows several, equivalent solutions to a particular problem. The intent of this approach is to address key concepts in dynamics and their application in solving problems. The student must use his/her own judgment in setting up and addressing the particulars of a problem.

Students learn how to address each module in Figure 1, each of which has specific outputs that require the application of basic concepts

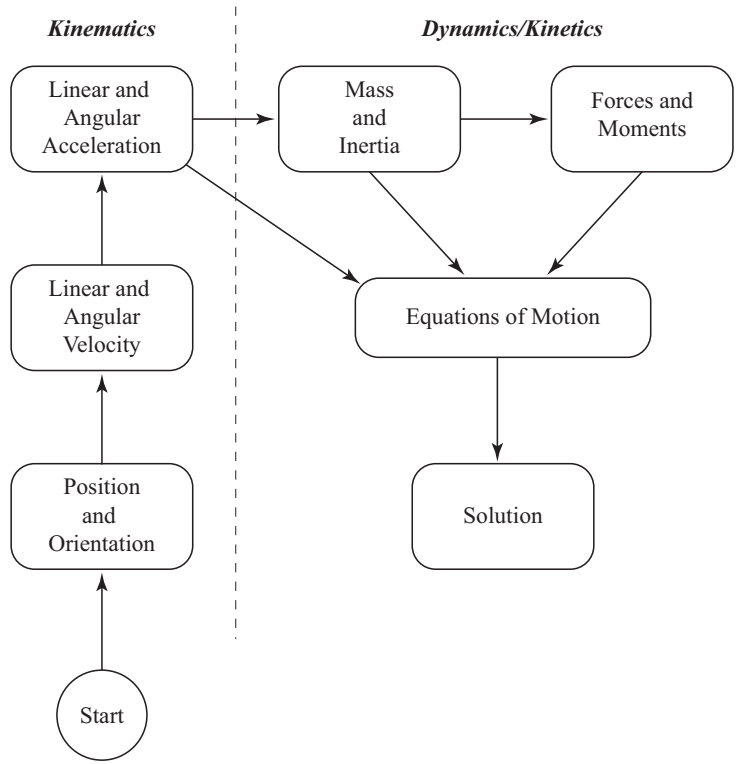

Figure 1: Problem Solving Process. in kinematics and dynamics. They eventually combine these outputs to obtain the equations of motion. It is easier to illustrate this structure using the simple example in Figure $2^{11}$. The problem statement is:

The double inclined plane supports two blocks $A$ and $B$, each having a weight of $10 \mathrm{lb}$. If the coefficient of kinetic friction between the blocks and the plane is $\mu_{k}=0.1$, determine the acceleration of each block. Assume the pulley is massless.

The next sections illustrate how students write up a solution to this problem. Each section represents a module in Figure 1. 


\subsection{Position and Orientation}

The first step in Figure 1, is to develop a description of position and orientation for all rigid bodies in the system. Students develop this description using points and directions, where three righthanded, orthogonal directions compose a reference frame. In the proposed process, points translate and directions rotate. A variable distance measures translation and a variable angle measures rotation. These variables com-

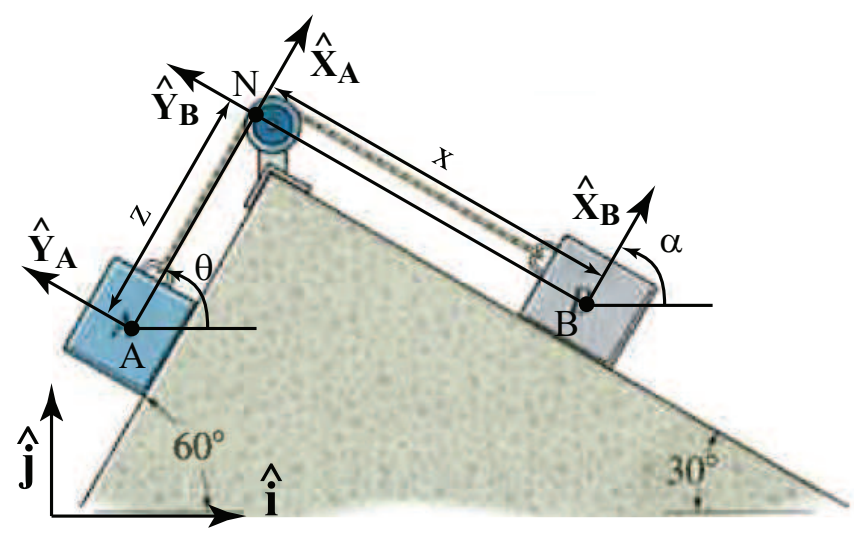

Figure 2: Coordinate System Diagram ${ }^{11}$. prise the generalized coordinates. The collection of generalized coordinates, points, and frames comprise a coordinate system, (this process involves other concepts, which this paper will not discuss in detail.) Each student, in turn, contributes to the development of the Coordinate System Diagram in Figure 2.

From Figure 2, students develop a location description for each rigid body in the system. The location description includes a position vector and a rotation matrix; however, this paper will not discuss these elements in detail. Students must provide the following write-up:

Simplifications: The pulley is massless.

Rigid Bodies: There are two rigid bodies to consider in this problem, the two blocks.

Inertial Reference Frame and Point: The inertial reference point is $\mathrm{N}$, and the inertial reference frame is $N=(\widehat{\mathbf{i}}, \widehat{\mathbf{j}})$.

Other Points and Frames: The body-attached points, A and B, represent the mass centers of each block. The body-attached frames are $\mathrm{A}=\left(\widehat{\mathbf{X}}_{\mathrm{A}}, \widehat{\mathbf{Y}}_{\mathrm{A}}\right)$ and $\mathrm{B}=\left(\widehat{\mathbf{X}}_{\mathrm{B}}, \widehat{\mathbf{Y}}_{\mathrm{B}}\right)$.

\section{Location Descriptions:}

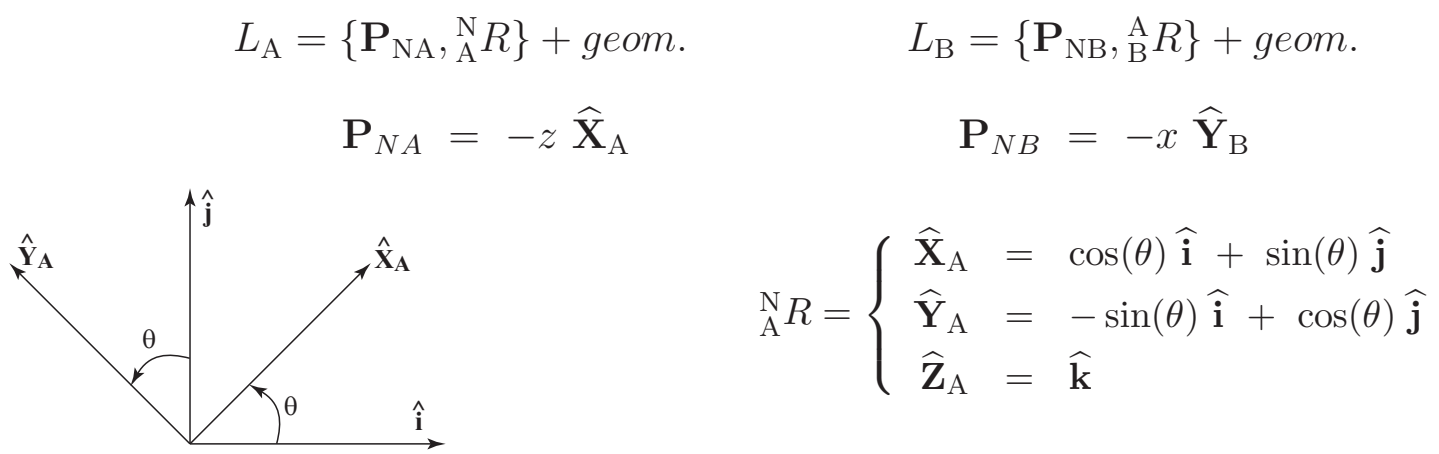




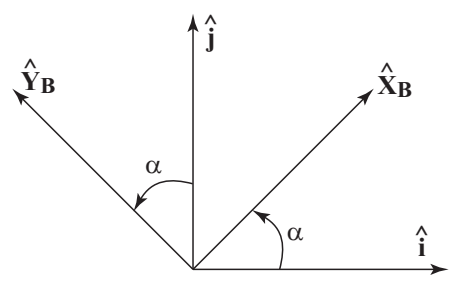

$$
{ }_{\mathrm{B}}^{\mathrm{N}} R=\left\{\begin{array}{l}
\widehat{\mathbf{X}}_{\mathrm{B}}=\cos (\alpha) \widehat{\mathbf{i}}+\sin (\alpha) \widehat{\mathbf{j}} \\
\widehat{\mathbf{Y}}_{\mathrm{B}}=-\sin (\alpha) \hat{\mathbf{i}}+\cos (\alpha) \hat{\mathbf{j}} \\
\widehat{\mathbf{Z}}_{\mathrm{B}}=\widehat{\mathbf{k}}
\end{array}\right.
$$

Students examine the location descriptions to determine the number of generalized coordinates/variables that are involved, and they write:

Coordinates: Examining the location descriptions shows that two coordinates, $x$ and $z$, can describe the motion of this system, (note that $\theta$ and $\alpha$ are constants.)

Students then imagine the motion of this system to determine whether their assigned coordinates are independent. Many problems in conventional textbooks include constrained systems, but give very little discussion of constraints. The discussion of constraints leads directly into one of degrees-of-freedom, which most textbooks barely discuss:

Constraints: Examining this system one can see that the two coordinates are not independent; fixing one fixes the other. To find the relationship, or constraint, between them, one must notice that the length of the rope must be constant:

$$
x+z=L=\text { constant }
$$

Degrees-of-Freedom (DOF): Therefore the system has one DOF,

$$
2 \text { coordinates }-1 \text { constraint }=1 \mathrm{DOF}
$$

The next step in solving the stated problem is to determine what the objectives are:

Givens: $m_{\mathrm{A}} g=m_{\mathrm{B}} g=10 l b, \mu_{k}=0.1, \theta(t)=60^{\circ}$, and $\alpha(t)=60^{\circ}$

Objectives: Find ${ }^{\mathrm{N}} \dot{\mathbf{V}}_{\mathrm{NA}}$ and ${ }^{\mathrm{N}} \dot{\mathbf{V}}_{\mathrm{NB}}$.

These objectives determine what needs to be done in all of the other modules in Figure 1. First of all, the objectives determine which velocities to find.

\subsection{Velocity}

In this section, the second block in Figure 1, students find the velocities they need to solve the problem. They find velocities by differentiating the position vectors and rotation expressions in the location descriptions. In class there are discussions of how to differentiate position vectors to obtain translational velocities, and how to find angular velocity; however, these discussions are too lengthy to present here. Because the blocks do not rotate, only their translational velocities are needed: 


$$
\begin{aligned}
& { }^{\mathrm{N}} \mathbf{V}_{\mathrm{NA}}=\frac{{ }^{\mathrm{N}} d \mathbf{P}_{\mathrm{NA}}}{d t}=\frac{d(-z)}{d t} \widehat{\mathbf{X}}_{\mathrm{A}}+(-z) \frac{{ }^{\mathrm{N}} d \widehat{\mathbf{X}}_{\mathrm{A}}}{d t}=-\dot{z} \widehat{\mathbf{X}}_{\mathrm{A}} \\
& { }^{\mathrm{N}} \mathbf{V}_{\mathrm{NB}}=\frac{{ }^{\mathrm{N}} d \mathbf{P}_{\mathrm{NB}}}{d t}=\frac{d(-x)}{d t} \widehat{\mathbf{Y}}_{\mathrm{B}}+(-x) \frac{{ }^{\mathrm{N}}}{d \widehat{\mathbf{Y}}_{\mathrm{B}}} d=-\dot{x} \widehat{\mathbf{Y}}_{\mathrm{B}}
\end{aligned}
$$

We can also differentiate the constraint equation to obtain the relationship between generalized speeds,

$$
\frac{d}{d t}(x+z=L) \quad \longrightarrow \quad \dot{x}+\dot{z}=0
$$

\subsection{Acceleration}

Students then find the accelerations they need to solve the problem, by differentiating the velocity vectors:

$$
\begin{aligned}
& { }^{\mathrm{N}} \dot{\mathbf{V}}_{\mathrm{NA}}=\frac{{ }^{\mathrm{N}} d^{\mathrm{N}} \mathbf{V}_{\mathrm{NA}}}{d t}=\frac{d(-\dot{z})}{d t} \widehat{\mathbf{X}}_{\mathrm{A}}+(-\dot{z}) \frac{{ }^{\mathrm{N}} d \widehat{\mathbf{X}}_{\mathrm{A}}}{d t}=-\ddot{z} \widehat{\mathbf{X}}_{\mathrm{A}} \\
& { }^{\mathrm{N}} \dot{\mathbf{V}}_{\mathrm{NB}}=\frac{{ }^{\mathrm{N}} d^{\mathrm{N}} \mathbf{V}_{\mathrm{NB}}}{d t}=\frac{d(-\dot{x})}{d t} \widehat{\mathbf{Y}}_{\mathrm{B}}+(-\dot{x}) \frac{{ }^{\mathrm{N}} d \widehat{\mathbf{Y}}_{\mathrm{B}}}{d t}=-\ddot{x} \widehat{\mathbf{Y}}_{\mathrm{B}}
\end{aligned}
$$

We can also differentiate the derivative of the constraint equation to obtain a relationship between accelerations as,

$$
\frac{d}{d t}(\dot{x}+\dot{z}=0) \quad \longrightarrow \quad \ddot{x}+\ddot{z}=0
$$

\subsection{Mass and Inertia}

The next step is to address mass and moments of inertia. Because the blocks do not rotate, this section is simple. The terms $m_{\mathrm{A}}$ and $m_{\mathrm{B}}$ designate the mass of blocks $\mathrm{A}$ and $\mathrm{B}$ respectively.

\subsection{Forces and Moments}

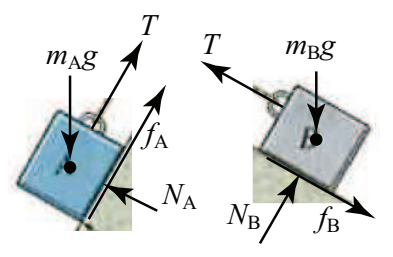

Figure 3: Free-Body Diagram.

The next step is to find the forces and moments on the blocks. This requires that students draw a Free-Body Diagram, see Figure 3 , which they learned in a prior course on statics. The sum of forces on body A is:

$$
\left(\sum \mathbf{F}\right)_{\mathrm{A}}=-m_{A} g \widehat{\mathbf{j}}+N_{A} \widehat{\mathbf{Y}}_{\mathrm{A}}+T \widehat{\mathbf{X}}_{\mathrm{A}}+f_{A} \widehat{\mathbf{X}}_{\mathrm{A}}
$$


Note that

$$
\widehat{\mathbf{j}}=\cos (\theta) \widehat{\mathbf{Y}}_{\mathrm{A}}+\sin (\theta) \widehat{\mathbf{X}}_{\mathrm{A}}
$$

therefore

$$
\left(\sum \mathbf{F}\right)_{\mathrm{A}}=-m_{A} g\left(\cos (\theta) \widehat{\mathbf{Y}}_{\mathrm{A}}+\sin (\theta) \widehat{\mathbf{X}}_{\mathrm{A}}\right)+N_{A} \widehat{\mathbf{Y}}_{\mathrm{A}}+T \widehat{\mathbf{X}}_{\mathrm{A}}+\mu_{k} N_{A} \widehat{\mathbf{X}}_{\mathrm{A}}
$$

The sum of forces on body B is:

$$
\left(\sum \mathbf{F}\right)_{\mathrm{B}}=-m_{B} g \widehat{\mathbf{j}}+N_{B} \widehat{\mathbf{X}}_{\mathrm{B}}+T \widehat{\mathbf{Y}}_{\mathrm{B}}-f_{B} \widehat{\mathbf{Y}}_{\mathrm{B}}
$$

Note that

$$
\widehat{\mathbf{j}}=\cos (\alpha) \widehat{\mathbf{Y}}_{\mathrm{B}}+\sin (\alpha) \widehat{\mathbf{X}}_{\mathrm{B}}
$$

therefore

$$
\left(\sum \mathbf{F}\right)_{\mathrm{B}}=-m_{B} g\left(\cos (\alpha) \widehat{\mathbf{Y}}_{\mathrm{B}}+\sin (\alpha) \widehat{\mathbf{X}}_{\mathrm{B}}\right)+N_{B} \widehat{\mathbf{X}}_{\mathrm{B}}+T \widehat{\mathbf{Y}}_{\mathrm{B}}-\mu_{k} N_{B} \widehat{\mathbf{Y}}_{\mathrm{B}}
$$

\subsection{Equations of Motion}

The previous sections addressed all of the components students need to form the equations of motion. Euler's first law shows how to combine these components into the equations of motion:

$$
\begin{gathered}
\left(\sum \mathbf{F}\right)_{\mathrm{A}}=m_{\mathrm{A}}{ }^{\mathrm{N}} \dot{\mathbf{V}}_{\mathrm{NA}} \\
-m_{\mathrm{A}} g\left(\cos (\theta) \widehat{\mathbf{Y}}_{\mathrm{A}}+\sin (\theta) \widehat{\mathbf{X}}_{\mathrm{A}}\right)+N_{A} \widehat{\mathbf{Y}}_{\mathrm{A}}+T \widehat{\mathbf{X}}_{\mathrm{A}}+\mu_{k} N_{A} \widehat{\mathbf{X}}_{\mathrm{A}}=-m_{\mathrm{A}} \ddot{z} \widehat{\mathbf{X}}_{\mathrm{A}} \\
\left(\sum \mathbf{F}\right)_{\mathrm{B}}=m_{\mathrm{B}}{ }^{\mathrm{N}} \dot{\mathbf{V}}_{\mathrm{NB}} \\
-m_{\mathrm{B}} g\left(\cos (\alpha) \widehat{\mathbf{Y}}_{\mathrm{B}}+\sin (\alpha) \widehat{\mathbf{X}}_{\mathrm{B}}\right)+N_{B} \widehat{\mathbf{X}}_{B}+T \widehat{\mathbf{Y}}_{\mathrm{B}}-\mu_{k} N_{B} \widehat{\mathbf{Y}}_{\mathrm{B}}=-m_{\mathrm{B}} \ddot{x} \widehat{\mathbf{Y}}_{\mathrm{B}}
\end{gathered}
$$

Separating these relations into scalar equations yields

$$
\begin{array}{ll}
-m_{\mathrm{A}} g \sin (\theta)+T+\mu_{k} N_{A}=-m_{\mathrm{A}} \ddot{z} & -m_{\mathrm{A}} g \cos (\theta)+N_{A}=0 \\
-m_{\mathrm{B}} g \cos (\alpha)+T-\mu_{k} N_{B}=-m_{\mathrm{B}} \ddot{x} & -m_{\mathrm{B}} g \sin (\alpha)+N_{B}=0
\end{array}
$$

Using the second derivative of the constraint equation and eliminating $N_{A}$ and $N_{B}$ yields two equations

$$
-m_{\mathrm{A}} g \sin (\theta)+T+\mu_{k} m_{\mathrm{A}} g \cos (\theta)=-m_{\mathrm{A}} \ddot{z}
$$




$$
-m_{\mathrm{B}} g \cos (\alpha)+T-\mu_{k} m_{\mathrm{B}} g \sin (\alpha)=m_{\mathrm{B}} \ddot{z}
$$

Eliminating $T$ yields the single equation of motion as

$$
g\left(m_{\mathrm{B}} \cos (\alpha)-m_{\mathrm{A}} \sin (\theta)\right)+\mu_{k} g\left(m_{\mathrm{A}} \cos (\theta)+m_{\mathrm{B}} \sin (\alpha)\right)=-\left(m_{\mathrm{A}}+m_{\mathrm{B}}\right) \ddot{z}
$$

\subsection{Solution}

Substituting the known parameters and givens into the equation of motion yields

$$
\begin{aligned}
10 l b\left(\cos \left(60^{\circ}\right)-\sin \left(60^{\circ}\right)\right)+(0.1)(10 l b)\left(\cos \left(60^{\circ}\right)+\sin \left(60^{\circ}\right)\right) & =-\frac{10 l b+10 l b}{32.2 f t / s^{2}} \ddot{z} \\
-2.2942 l b & =-\frac{20 l b}{32.2 f t / s^{2}} \ddot{z} \quad \rightarrow \quad \ddot{z}=3.69 \mathrm{ft} / \mathrm{s}^{2}
\end{aligned}
$$

From the second derivative of the constraint equation we obtain

$$
\ddot{x}=-3.69 \mathrm{ft} / \mathrm{s}^{2}
$$

therefore

$$
{ }^{\mathrm{N}} \dot{\mathbf{V}}_{\mathrm{NA}}=-3.69 f t / s^{2} \widehat{\mathbf{X}}_{A} \quad{ }^{\mathrm{N}} \dot{\mathbf{V}}_{\mathrm{NB}}=3.69 f t / s^{2} \widehat{\mathbf{Y}}_{B}
$$

\section{Instructional Approach: Direct Questioning}

The write-up in Section 2 suggests a pattern of steps that a student can learn to enable their analysis of a system of rigid bodies in motion, which may include some flexible bodies like ropes. There is an order and sequence to the write-up corresponding to the modules in Figure 1. Therefore, if the instructor poses a problem in class, the students should know the procedure to follow. Different problems will yield different results, but the structure and process remain the same.

Consider each expression or diagram in the write-up given in Section 2 as a single step in the process. Each expression or part of a diagram exemplifies the application of one or two simple concepts. In fact, the effort in Section 2 is to divide the analysis into simple steps whose application does not require much intuition or mental leaps, even though the steps are not trivial.

With this structure, the instructor can work through the class, student by student, asking each one to contribute to the next small step in the problem. The instructor writes down the response on the board, even if it is incorrect, so that the other students may examine the response. It is not difficult to work through the entire class having each student add to 
the problem's solution. It is common that in a class of 60 students in a 1.5 hour period, the instructor can question each student twice.

The author has used this approach in his graduate and undergraduate dynamics courses over the last four years and has observed several benefits. The undergraduate dynamics course is the first one taken during the first semester of the sophomore year. At this point, all of the observations presented in the paper are anecdotal, but the authors desire to investigate this technique in a more formal manner in the future. The next sections discuss the current observations in detail by identifying three themes of interest: student engagement, affective pedagogy issues, and class management and assessment.

\subsection{Student Engagement}

A large body of work exists examining student engagement and learning at the college level $^{24 ; 10}$. Student engagement is a multidimensional phenomenon including at least some behavioral and affective components. It significantly improves students' ability to learn course concepts and recall information ${ }^{22}$. While many argue that engagement and motivation are rooted in individual cognitive skills, several authors recognize the importance of classroom experience in shaping one's dispositions toward education and learning.

The proposed technique stems from ideas in active and participative learning, which have been proposed as methods for increasing student engagement. This type of learning includes techniques such as speaking activities, discussions, case-studies, concept tests, anonymous voting, and problem-solving ${ }^{12}$. Students are able to better recall the lecture content when the instructor uses interactive periods or pauses between topics ${ }^{13 ; 31}$. In particular, it was found that instructor-led discussion improved performance on short answer exam sections, while role-play sessions did so on multiple choice exam sections ${ }^{27}$. Other successful methods include collaborative learning 7;8;4;33;2, cooperative learning 6;26;32;14;16;15, and problem-based learning $3 ; 25 ; 36 ; 1 ; 18$. Many other works champion the effectiveness of active and engagement-based learning methods ${ }^{38 ; 9 ; 29 ; 21 ; 17 ; 28}$. Some disadvantages of interactive lectures include reduction in content, loss of control, and student resistance ${ }^{35 ; 34 ; 19 ; 20 ; 23 ; 13 ; 30}$. The active learning technique similar to the one proposed in this paper is problem based learning (PBL). This approach requires students to learn by solving real-world problems, rather than sitting passively in a lecture ${ }^{39}$.

In the proposed approach, the instructor asks every student in the class a question. Several students have remarked that this keeps them awake and paying attention in class. The questioning usually starts on the first row and simply moves from one student to the next, in seating order, along each row from the front to the back row of the class. The instructor asks each student a short question, such as "What's next?" This question does not hint at what the next step is; however, the instructor can alter the question to provide a hint if s/he wants. Thus the students must keep track of the solution's progress in order to answer correctly. They must also know the structure given in Section 2 and keep track of the progress toward a solution relative to that structure. This keeps students engaged even if the 
instructor is not questioning them at the moment. Since the instructor records all responses, even the incorrect ones, students can learn about possible pitfalls. They can also observe how the correct response is shaped and the problem solving is progressing. Therefore, students are engaged not only in solving the problem, but in developing some judgment in how to apply the process.

In addition, at any time any student in the class may object to another student's answer, which increases the level of attention and engagement. The proposed process allows for many different solutions to a particular problem. Some students may think of a simpler way to analyze the problem and can redirect the solution process if they want. This involves a brief discussion, a vote, and if most students want the change, the new direction is adopted. Being cognizant of an objection requires the student to follow the current state of the solution, but also to search for a better one. This keeps engaged students whom the instructor is not currently questioning.

If a student whose turn it is to contribute does not know what to do next, s/he "punts" rather than spend a lot of time trying to guess or figure it out. The instructor does not attempt to provide hints or coaching to lead the student to the next step, because this slows down the process and the other students will disengage. Quickly moving on keeps subsequent students on their toes, rather than having all students watch an extensive coaching session with a single student. Approximately $95 \%$ of the time, there is a subsequent student who knows the next step. However, if no one in class can tell the next step, it is a sign that the instructor needs to spend some time discussing the next step and/or reviewing concepts.

In addition, if a student is struggling or taking a long time to answer, the instructor should punt the student. The student who punts, or is punted by the instructor, has a significant interest in hearing the correct next step, which keeps him/her engaged even though they chose to punt. For this to happen, the goal is to keep the questioning and answering moving through the class to reach as many students as possible and to quickly discover the correct answer.

Also note that there is an order to applying the process, as the flow chart in Figure 1 suggests. If a student attempts to jump too far ahead, or skip steps, the instructor should punt or correct the student. It depends on which is faster. If it takes a long explanation to tell him/her why his/her answer skips a step, then punt the student. If the explanation is short, the instructor can provide it and give the student more time to come up with the answer. Again the goal is to keep the process moving from student to student, not to give a long coaching session to a particular student.

\subsection{Affective Pedagogy Issue}

Affective pedagogy refers to aspects of teaching and the classroom environment that affect the mood, feelings, or attitudes of the students toward learning. The proposed approach has a significant effect in these areas, the key one having to do with punting, since it clearly 
shows that some students do not know the answer or do not follow the sequence of steps. Having to punt, or being punted, can have a positive or negative effect on the students. The positive aspect is that they see that not everyone knows all of the answers, which gives some comfort to many students. It removes the silent fear that they may be the only person who is not getting it. This can remove feelings of inferiority that can grip a student, leaving them mute and desperate in a difficult class like dynamics. The authors feel that overcoming an inferiority complex helps in building one's self-confidence, and this is one of the greatest benefits of this approach.

The instructor is responsible for mitigating the negative aspects of direct questioning, such as embarrassment. The authors never expect that 100\% of the students will find this approach interesting and fun all of the time, but it does not have to be traumatizing. The instructor's attitude can make a tremendous difference. The intent is that the atmosphere should be more light-hearted than punitive. The instructor's behavior should not give the impression that punting is an admission of stupidity, lack of effort, or inferiority. It is simply an acknowledgment that the student does not know the next step in the analysis of a particular problem at a particular time. This is also the reason for not dwelling on a single student. Attempting to coach a student toward the correct answer may only amplify their embarrassment. Quickly move on and the students take it lightly. They will also move on and focus on the next step. As a result, students become more interested in the progress of the problem's solution.

If the instructor maintains a positive attitude toward the students, this approach takes on a game-like atmosphere that most students seem to enjoy. Several students have commented, even during the course, that the dynamics course is or was their favorite class. Imagine students enjoying learning dynamics. Maintaining a positive atmosphere in the class where there is no pressure to know everything at all times, makes the classroom a safe environment where students can freely make mistakes in their effort to learn the material. They ask more questions. They participate more freely. They try harder. They attempt to teach their friends. The approach works quite well in creating a positive attitude toward dynamics, even though most students enter with a great deal of apprehension about this "weed-out" class. It is unrealistic to expect $100 \%$ success, but the overall response to this approach by the students has been significantly and noticeably positive.

\subsection{Instructor Class Management and Assessment}

The application of this approach should result in a game-like atmosphere much like the many online multi-user gaming applications that many students play. The instructor and students can both have fun with it. It is necessary that the instructor becomes comfortable with the noise level and slightly out-of-control nature of this exercise. There are typically several side-conversations in progress as students ask their peers explain parts of the problem, or attempt to obtain the correct next step from their peers. Although students try to do this quietly, there is usually an underlying murmur as the session goes on. Typically, students 
are trying to keep track of the solution's progress, so the background noise remains low. If the instructor considers this murmur to indicate positive participation, typically students teaching students, then there is no need to stop it. The authors consider this low-level hum to be a good thing that demonstrates that students are engaged.

Some students may begin joking around during this game. It is also unnecessary to stop this, unless it becomes a distraction. This is another situation where it is important to keep the questions and answers moving, without focusing on any one student or giving others a chance to "perform." It is tiresome for the entire class to watch while one student attempts to make the classroom an audition for a comedy club. This behavior can lighten the mood for a while as long as the instructor does not dwell on it and keeps the questioning and answering moving along from one student to the next. Thus, this approach may not work well for an instructor that has a strong desire to strictly control every aspect of in-class interaction or is distracted by the chaotic intervention of some students.

After a couple of classes when this exercise is repeated during a semester, the instructor will gain a feel for the progress of the students in the class. Because the questions are nonleading and non-coaching, the student's response gives a great deal of information about how or whether they are following the material. The instructor can easily identify students who experience more difficulties with the course material, and can thus intervene (e.g., maybe suggest that particular students visit office hours), to keep students from falling too far behind.

\section{Conclusion}

The observations presented in this paper suggest that the proposed approach is highly effective for teaching an undergraduate dynamics course. First, it uses a highly structured problem solving approach that shows how basic concepts in dynamics can be applied to different problems. The second key aspect is a direct questioning approach that keeps all students in the class engaged in problem-solving. The authors experience infers that this approach will increase learning in one of the more difficult areas in undergraduate engineering education. This should lead to greater retention of students in many of the engineering disciplines. Moreover, this approach can contribute to enhancing student engagement with the subject. However, the evidence that attests to the efficacy of this approach is based on observations by the authors, not on a systematic examination. The next goal in the devel-

opment of this approach is to devise assessment methods that can more formally validate the assertions made herein.

\section{References}

[1] Mark A Albanese and Susan Mitchell. Problem-based learning: A review of literature on its outcomes and implementation issues. Academic medicine, 68(1):52-81, 1993. 
[2] Richard Anderson, Ruth Anderson, Oliver Chung, KM Davis, Peter Davis, Craig Prince, Valentin Razmov, and Beth Simon. Classroom presenter: a classroom interaction system for active and collaborative learning, 2006.

[3] Howard S Barrows. Problem-based learning: An approach to medical education. Springer Publishing Company, 1980.

[4] Lemuel Berry Jr. Collaborative learning: A program for improving the retention of minority students., 1991.

[5] Alan P. Bowling. Vector Mechanics: A Systematic Approach. Aqualan Press, LLC, first edition, 2015.

[6] Kenneth A Bruffee. Sharing our toys: Cooperative learning versus collaborative learning. Change: The Magazine of Higher Learning, 27(1):12-18, 1995.

[7] Pierre Dillenbourg et al. Collaborative-learning: Cognitive and computational approaches. Technical report, Elsevier, 1999.

[8] Elaine Fredericksen. Minority students and the learning community experience: A cluster experiment., 1998.

[9] Richard R Hake. Interactive-engagement versus traditional methods: A six-thousand-student survey of mechanics test data for introductory physics courses. American journal of Physics, 66(1):64-74, 1998.

[10] M. M. Handelsman, W. L. Briggs, N. Sullivan, and A. Towler. A measure of college student course engagement. The Journal of Educational Research, 98(3):184-191, 2005.

[11] R. C. Hibbeler. Engineering Mechanics: Dynamics. Pearson Prentice Hall, Upper Saddle River, New Jersey, 13 edition, 2013.

[12] James E Houston. Thesaurus of ERIC descriptors. Oryx Press, 13th edition edition, 1995.

[13] Mark Huxham. Learning in lectures: Do interactive windows help? Active Learning in Higher Education, 6(1):17-31, 2005.

[14] David W Johnson et al. Cooperative Learning: Increasing College Faculty Instructional Productivity. ASHE-ERIC Higher Education Report No. 4, 1991. ERIC, 1991.

[15] David W Johnson, Roger T Johnson, and Karl A Smith. Cooperative learning returns to college what evidence is there that it works? Change: the magazine of higher learning, 30(4):26-35, 1998.

[16] David W Johnson, Roger T Johnson, and Karl Aldrich Smith. Active learning: Cooperation in the college classroom. Interaction Book Company Edina, MN, 1991.

[17] Marcia W Keyser. Active learning and cooperative learning: understanding the difference and using both styles effectively. Research Strategies, 17(1):35 - 44, 2000.

[18] R. Lacuesta, G. Palacios, and L. Fernandez. Active learning through problem based learning methodology in engineering education. In Frontiers in Education Conference, 2009. FIE '09. 39th IEEE, pages $1-6$, Oct 2009.

[19] David A Lake. Student performance and perceptions of a lecture-based course compared with the same course utilizing group discussion. Physical Therapy, 81(3):896-902, 2001.

[20] William J Lammers and John J Murphy. A profile of teaching techniques used in the university classroom a descriptive profile of a us public university. Active Learning in Higher Education, 3(1):54-67, 2002. 
[21] Priscilla Laws, David Sokoloff, and Ronald Thornton. Promoting active learning using the results of physics education research. UniServe Science News, 13:14-19, 1999.

[22] Jay McTighe and Grant Wiggins. Understanding by design. Association for Supervision and Curriculum Development (ASCD), 1998.

[23] R Murray and JR Brightman. Interactive teaching. European journal of engineering education, 21(3):295-308, 1996.

[24] National Survey of Student Engagement (NSSE). Exploring different dimensions of student engagement. Technical report, Indiana University Center for Postsecondary Research, Bloomington, IN, 2005.

[25] Geoffrey R Norman and Henk G Schmidt. Effectiveness of problem-based learning curricula: theory, practice and paper darts. Medical education, 34(9):721-728, 2000.

[26] Theodore Panitz. Collaborative versus cooperative learning: A comparison of the two concepts which will help us understand the underlying nature of interactive learning., 1999.

[27] Leanne C. Powner and Michelle G. Allendoerfer. Evaluating hypotheses about active learning. International Studies Perspectives, 9(1):75-89, 2008.

[28] Michael Prince. Does active learning work? a review of the research. Journal of Engineering Education, 93(3):223 - 231, 2004.

[29] Edward F Redish, Jeffery M Saul, and Richard N Steinberg. On the effectiveness of active-engagement microcomputer-based laboratories. American Journal of Physics, 65(1):45-54, 1997.

[30] Donna Riley and Lionel Claris. From persistence to resistance: Pedagogies of liberation for inclusive science and engineering. International Journal of Gender, Science and Technology, 1(1), 2009.

[31] Kathy L. Ruhl, Charles A. Hughes, and Patrick J. Schloss. Using the pause procedure to enhance lecture recall. Teacher Education and Special Education: The Journal of the Teacher Education Division of the Council for Exceptional Children, 10(1):14-18, 1987.

[32] Robert E Slavin. Cooperative learning. Review of educational research, 50(2):315-342, 1980.

[33] Leonard Springer, Mary Elizabeth Stanne, and Samuel S Donovan. Effects of small-group learning on undergraduates in science, mathematics, engineering, and technology: A meta-analysis. Review of educational research, 69(1):21-51, 1999.

[34] L. A. Van Dijk, G. C. Van Der Berg, and H. Van Keulen. Interactive lectures in engineering education. European Journal of Engineering Education, 26(1):15-28, 2001.

[35] Liesbet A van Dijk, Gerard C van den Berg, and Hanno van Keulen. Using active instructional methods in lectures: A matter of skills and preferences. Innovations in Education 8 Training International, 36(4):260-272, 1999.

[36] David T Vernon and Robert L Blake. Does problem-based learning work? a meta-analysis of evaluative research. Academic medicine, 68(7):550-63, 1993.

[37] Debby R. Walser-Kuntz, Sarah E. Deel, and Susan R. Singer. Faculty-coached, in-class problem solving. http://serc.carleton.edu/sp/library/coached_problems/index.html. last modified June 3, 2015.

[38] Susan Welch. Nursing caps to feminist pedagogy: transformation of nursing education. Teaching and Learning in Nursing, 6:102-108, 2011. 
[39] Aman Yadav, Dipendra Subedi, Mary A. Lundeberg, and Charles F. Bunting. Problem-based learning: Influence on students' learning in an electrical engineering course. Journal of Engineering Education, 100(2):253-280, 2011. 